\title{
Volatile lunacy
}

\author{
Over the past six years an increasingly complex view of water inside and on the surface of the Moon \\ has emerged. Lunar water has moistened sticky questions, and so renewed lunar exploration efforts \\ are needed to deepen our knowledge of the Earth-Moon system.
}

Only six years ago, much of what we thought we knew about the Moon suddenly seemed doubtful. A significant quantity of water molecules, trapped within small beads of volcanic glass in Apollo samples ${ }^{1}$, seemed irreconcilable with a body born from the hot debris of a giant impact, without enough gravity to hold an atmosphere and with a surface exposed to a harsh space weathering environment. Unlike rocks found on Earth, early analyses of the Apollo samples did not reveal any water-bearing minerals, or any evidence for aqueous alteration. For decades, lunar scientists assumed that the Moon was dry. As discussed in a web focus published with this issue (http://www.nature.com/ngeo/ focus/lunar-water/index.html), the 2008 discovery of lunar water was not an isolated case. And the presence of water, even in sparse amounts, has the potential to stir up lunar science.

Modern analytical techniques, applied to the decades-old lunar sample collection, have also revealed appreciable amounts of water in apatite minerals from vast basaltic plains that arise from deep mantle sources, as well as in other lunar crustal rocks thought to be later-stage magmatic intrusions. And water bound to minerals that formed in the lunar interior and were then exposed by impacts has been remotely sensed from orbit ${ }^{2}$. As Robinson and Taylor demonstrate in a Review Article on page 401, the emerging picture of water in the Moon's interior is one of heterogeneity. Lunar models face a daunting challenge to explain a Moon within which wetness varies.

Lunar water has been detected as molecules locked up within rocks' mineral structures, and not as pools of liquid as on Earth, but there are perplexing similarities to the Earth's interior. Although the water content of the volcanic glasses - a few parts per million - was enough to dampen the notion of a bone-dry Moon, the glasses would have lost water during eruption, and the source regions in the lunar mantle may contain as much water as mid-ocean ridge basalts on Earth ${ }^{1}$. Mid-ocean ridge basalts are relatively dry, so this does not mean that the Moon contains as much water as

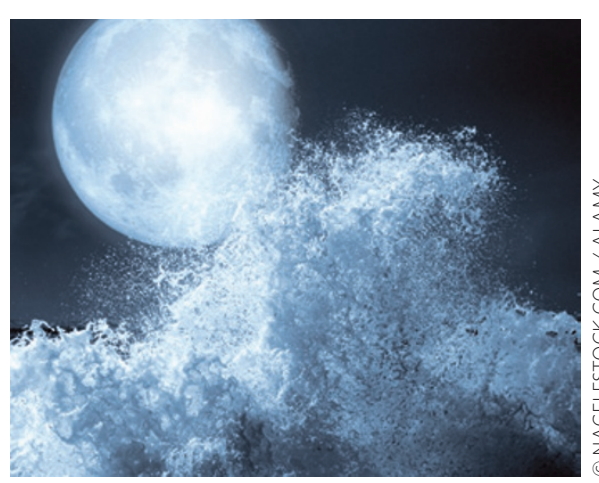

Just one month later, the hypothesis was confirmed when a spent Centaur rocket disconnected from NASA's Lunar Crater Observation and Sensing Satellite (LCROSS) spacecraft and deliberately crashed into a permanently shadowed crater. Within the plume of material thrown up by the impact, the LCROSS spectrometer detected about 150 kilograms of water ${ }^{5}$. The water ice stored in polar regions could have been implanted in the lunar regolith by the solar wind ${ }^{6}$ or delivered by meteorite or cometary impacts.

The discoveries of water inside and Earth, but the retention of so much water in the lunar interior is more than can be accommodated easily by many Moon formation models ${ }^{3}$.

Volatile elements are a central constraint in our understanding of planet formation and evolution. For example, the apparent dearth of water on the Moon and the abundance of it on Earth was one line of evidence for the Moon's formation in a giant impact. Furthermore, as we know from terrestrial studies, volatile elements affect mantle dynamics, volcanism, seismicity and other geological processes. For example, there is evidence that the ancient Moon had a complex lunar dynamo (page 409), the enigmatic longevity of which could be attributed to a waterenriched reservoir near the core-mantle boundary (page 400).

The discovery of lunar water has also triggered a reassessment of surface processes. A year after the discovery of water bound within lunar igneous rocks, traces of water were remotely detected across the lunar surface, especially at high latitudes. In September 2009, NASA reported that its Moon Mineralogy Mapper spectrometer aboard the Indian Space Research Organization's Chandrayaan-1 spacecraft had detected absorption features consistent with water or hydroxyl ${ }^{4}$, a result supported by detections from the Deep Impact and Cassini spacecraft. This indicated that active surface processes were forming and preserving water and hydroxyl on the Moon, and suggested that permanently shadowed polar regions could host water ice reservoirs. on the surface of the Moon are fuelling renewed passion for lunar exploration in an increasingly diverse crowd. As the Chinese Chang'e 3 spacecraft representing the first soft landing on the Moon since 1976 - and its rover Yutu sit on the lunar surface studying the surrounding terrain (page 391), privately funded teams across the world are scrambling for the $\$ 30$ million Google Lunar XPRIZE, promised to the first team with a spacecraft that lands safely on the lunar surface, travels 500 metres and beams images back to Earth ${ }^{7}$. And the Shackleton Energy company in Texas, USA, plans to mine the Moon's surface water to fuel space exploration ${ }^{8}$.

'Follow the water' has long been NASA's motto for Mars exploration'. Unlike on Mars, it is doubtful that the Moon's water ever existed in liquid form, let alone supported life. But, as is the case for Mars ${ }^{10}$, deciphering the presence of lunar water with targeted exploration efforts can help to unravel the formation and evolution of the Moon, and by extension its companion from birth, the Earth.

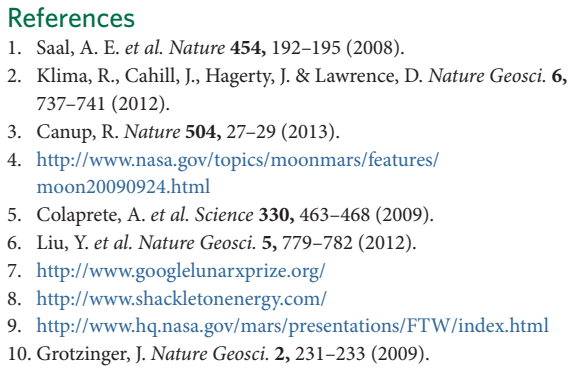

